\title{
Stratification of the space of unimodal interval maps
}

\author{
LOUIS BLOCK AND DAVID HART \\ Department of Mathematics, University of Florida, Gainesville, Florida 32611, USA
}

(Received 12 January 1983 and revised 20 May 1983)

\begin{abstract}
The space of 'quadratic-like' (unimodal) maps of a compact interval to itself is shown to decompose in a 'nice' way (stratify) according to a dynamical property of such maps (the existence of a homoclinic periodic orbit with given period). This decomposition is refined by that discovered by Sarkovskii. Orbit structure and bifurcation properties are also discussed.
\end{abstract}

\section{Introduction}

This paper discusses the space of maps of a compact interval and its decomposition according to their dynamical properties. Recall that if $F(n)$ denotes those maps in $C^{0}(I, I)$ (the continuous functions $f:[0,1] \rightarrow[0,1]$ ) having a point of least period $n$, then $F(j) \supset F(k)$ if and only if $j$ precedes $k$ in Sarkovskii's order [13]:

$$
\begin{aligned}
& 1 \Delta 2 \Delta 4 \Delta 8 \Delta \cdots \\
& \cdots \Delta 2^{2} \cdot 9 \Delta 2^{2} \cdot 7 \Delta 2^{2} \cdot 5 \Delta 2^{2} \cdot 3 \Delta \\
& \cdots \Delta 2 \cdot 5 \Delta 2 \cdot 3 \Delta \cdots \Delta 5 \Delta 3 .
\end{aligned}
$$

Moreover, in $C^{1}(I, I), j \Delta k$ implies that $\mathrm{cl} F(k) \subset$ int $F(j)$ [3], [4]; we use the term 'stratification' to denote such a structure. We remark that $F(j)$ is closed if $j$ is not a power of two. Also, 'infinity' may be inserted after the powers of two but before the other integers, to denote those maps with all powers of two as periods; for all $r \geq 0$ and odd $m>1, F\left(2^{r}\right) \supset F(\infty) \supset F\left(m \cdot 2^{r}\right)$, and $F(\infty)$ is closed.

A map has a point of period not a power of two if and only if it has a homoclinic point, if and only if it has positive topological entropy [1], [11]. Denote those maps which have a homoclinic point of period $n$ by $H(n)$. In general, the relationship between these sets, or between them and the Sarkovskii stratification, is not particularly elegant [5]. If one considers the class $\mathscr{C}$ (defined in $\S 3$ below) of 'quadraticlike' maps, however, things become quite tidy.

THEOREM. In $\mathscr{C}, F\left(m \cdot 2^{r}\right) \subset H\left(2^{r}\right)$, for any $r \geq 0$ and odd $m>1$. Moreover, $H\left(2^{r}\right)$ is a closed subset and $H\left(2^{r}\right) \backslash \bigcup_{m} F\left(m \cdot 2^{r}\right)$ is the boundary of $\bigcup_{m} F\left(m \cdot 2^{r}\right)$, for $r$ and $m$ as before. 
This gives rise to the following structure, where all sets are relative to $\mathscr{C}$.

$$
\begin{aligned}
& F(1) \supset F(2) \supset F(4) \supset \cdots \supset F(\infty) \supset \\
& \vdots \\
& H\left(2^{2}\right) \supset \cdots \supset F\left(5 \cdot 2^{2}\right) \supset F\left(3 \cdot 2^{2}\right) \supset \\
& H(2) \supset \cdots \supset F(5 \cdot 2) \supset F(3 \cdot 2) \supset \\
& H(1) \supset \cdots \supset F(5) \supset F(3) .
\end{aligned}
$$

The maps only in the first row are exactly those with zero entropy; the maps with positive entropy are stratified by the homoclinic sets, for powers of two, which in turn are stratified by the corresponding Sarkovskii sets. This structure yields immediate information on the bifurcations which must occur for a family in $\mathscr{C}$, as in [4], [5]. In particular, an arc $f_{t}$ from $F(1) \backslash F(2)$ to $F(3)$ passes through the $H\left(2^{r}\right)$ in the order indicated, by a sequence of homoclinic bifurcations (separated by sequences of periodic bifurcations).

In proving these results, we also establish the following, where 'simple periodic orbit' is defined as in [2], [8], [15] (and also in $\$ 2$ below).

THEOREM. Any map in $C^{0}(I, I)$ which has a periodic point of period $n$ has a simple orbit of period $n$.

For unimodal maps this may be obtained via kneading sequences, as in [9]; the authors thank the referee for this observation and other helpful comments.

\section{Simple periodic orbits}

We first show that it suffices to consider a particular type of orbit structure.

Definition. Let $S=\left\{x_{1}, \ldots, x_{n}\right\}$ be a finite set of points on the interval $I=[0,1]$ with $x_{1}<x_{2}<\cdots<x_{n}$. We say $S$ is separated to order 1 under $f$ if $n$ is even, $n=2 m$, and the sets $S_{1}=\left\{x_{1}, \ldots, x_{m}\right\}$ and $S_{2}=\left\{x_{m+1}, \ldots, x_{n}\right\}$ are interchanged by $f$. We say $S$ is separated to order $r$ under $f$ if $S$ is separated to order 1 and each of the sets $S_{1}$ and $S_{2}$ is separated to order $r-1$ under $f^{2}$.

Definition. Let $P$ be a periodic orbit of $f$, consisting of $m \cdot 2^{r}$ points, where $m$ is odd. We say $P$ is simple if the following conditions hold:

(i) if $r>0, P$ is separated to order $r$ under $f$, and

(ii) if $m>1, P=P_{1} \cup P_{2} \cup \cdots \cup P_{\left(2^{r}\right)}$, where $P_{1}$ denotes the least $m$ points of $P$, $P_{2}$ the next $m$ points, etc., and each $P_{k}$ is periodic for $f^{\left(2^{r}\right)}$ of Stefan type [15], i.e. $P_{k}=\left\{x_{1}, x_{2}, \ldots, x_{m}\right\}$ where

$$
f^{\left(2^{r}\right)}\left(x_{i}\right)=x_{i+1}
$$

and either

$$
x_{m}<x_{m-2}<\cdots<x_{1}<x_{2}<\cdots<x_{m-1}
$$

or the opposite order.

Note that if $P$ as above is simple, the sets $P_{1}, P_{2}, \ldots, P_{\left(2^{\prime}\right)}$ are permuted by $f$. We denote the maps with a simple periodic orbit of period $n$ by $F_{s}(n)$. 
We also require the following notion: the Markov graph of a set $\left\{p_{i}\right\}$, where $p_{i}<p_{i+1}$, is the directed graph whose vertices are the intervals $I_{j}=\left[p_{j}, p_{j+1}\right]$, possibly renumbered, with an arrow $I_{j} \rightarrow I_{k}$ if and only if $f\left(I_{j}\right) \supset I_{k}$.

LEMMA [6]. If a Markov graph of $f$ contains a loop $I_{0} \rightarrow I_{1} \rightarrow \cdots \rightarrow I_{n-1} \rightarrow I_{0}$, there is a point $x \in I_{0}$ with $f^{k}(x) \in I_{k}$, and $f^{n}(x)=x$.

The main result of this section generalizes [2], [8], [15].

THEOREM A. If $f \in C^{0}(I, I)$ has a periodic orbit of period $n$, then $f$ has a simple periodic orbit of period $n$ :

$$
F(n)=F_{s}(n) \text {. }
$$

The proof of theorem A is by 'induction' using the Sarkovskii order; we break it into seven steps, some of which are already known. The first step is to note that if all periods of $f$ are powers of two, then each orbit of $f$ is simple. This follows from [2].

We next show that an orbit of maximal period (in the sense of Sarkovskii) is simple. This follows from [8]; we give a much shortened proof. Consider such an orbit, of period $m \cdot 2^{r}$, for odd $m>1$ and $r>0$ (we may assume $m>1$, by the first step, and $r>0$, by [15]). As in [6], some vertex $I_{1}$ of the usual Markov graph associated to this orbit covers itself, $f\left(I_{1}\right) \supset I_{1}$, and there is a path

$$
I_{1} \rightarrow I_{2} \rightarrow \cdots \rightarrow I_{k}, \quad \text { for each } k \leq m \cdot 2^{r},
$$

but there is no edge $I_{k} \rightarrow I_{1}$ (or there would be a loop of odd length, and a periodic point of odd period, contradicting the Sarkovskii-maximality of $m \cdot 2^{r}$ ). But then all points in the orbit which are to the left of $I_{1}$ move to the right, and vice versa, so the orbit is separated to order one. Each half of the orbit is now a complete periodic orbit for $f^{2}$, of period $m \cdot 2^{r-1}$, and by induction the argument is completed.

The third step is that if $m>1$ is odd and $f \in F_{s}(m)$, then $f \in F_{s}(k)$ for any odd $k>m$. By hypothesis, there is an orbit $\left\{p_{i}\right\}, 1 \leq i \leq m$, with $f\left(p_{i}\right)=p_{i+1}$, and lying either in the order

$$
p_{m}<\cdots<p_{3}<p_{1}<p_{2}<\cdots<p_{m-1}
$$

or in the opposite order; (assume that given, for definiteness). There is a fixed point $e \in\left(p_{1}, p_{2}\right)$, and $x \in\left(e, p_{2}\right)$ with $f(x)=p_{1}$, also $y \in\left(p_{1}, e\right)$ with $f(y)=x$. Let $I_{1}, \ldots, I_{m-1}$ be the intervals defined above with

$$
I_{1}=\left[p_{1}, p_{2}\right], \quad I_{2}=\left[p_{3}, p_{1}\right], \quad I_{3}=\left[p_{2}, p_{4}\right], \text { etc. }
$$

We form a new Markov graph with

$$
J_{1}=[y, e], \quad J_{2}=[e, x], \quad J_{3}=\left[p_{1}, y\right], \quad J_{4}=\left[x, p_{2}\right],
$$

and

$$
J_{k}=I_{k-3} \quad \text { for } 5 \leq k \leq m+1 .
$$

There is, by the lemma above, a point $w \in J_{1}$, fixed by $f^{m+2}$, with $f^{k}(w) \in J_{k+1}$; this gives a simple orbit of period $m+2$. The assertion follows by induction. 
The fourth step is $F_{s}\left(m \cdot 2^{r}\right) \subset F_{s}\left(k \cdot 2^{r}\right)$, for any $r$, with $k, m$ as above. We denote the first (leftmost) $m$ points in the orbit of length $m \cdot 2^{r}$ by $P_{1}$, etc; $P_{\left(2^{r}\right)}$ denotes the last $m$ points. By hypothesis, $f$ permutes $P_{1}, P_{2}, \ldots, P_{\left(2^{\prime}\right)}$, and for each $i, P_{i}$ is a simple periodic orbit of $f^{\left(2^{r}\right)}$ of period $m$. In each $P_{i}$ we can choose points $x_{i}$ and $y_{i}$ so that the proof of step three can be applied to $f^{\left(2^{\prime}\right)}$ and the orbit $P_{i}$. The resulting orbit is a simple periodic orbit of $f$ of period

$$
m \cdot 2^{r}+2 \cdot 2^{r}=(m+2) \cdot 2^{r} \text {. }
$$

Again the conclusion follows by induction.

Fifth, $F_{s}(m) \subset F_{s}(6)$ for odd $m \geq 1$. Let $\left\{p_{1}, \ldots, p_{m}\right\}$ be as before; by step two we may assume $m \geq 9$. If $\left\{I_{k}\right\}$ is the Markov graph associated to this orbit in step two, it contains the loop

$$
I_{m-6} \rightarrow I_{m-5} \rightarrow \cdots \rightarrow I_{m-1} \rightarrow I_{m-6}
$$

since it is simple (as in [6]); hence there is a simple orbit of period six.

Sixth, $F_{s}\left(m \cdot 2^{r}\right) \subset F_{s}\left(3 \cdot 2^{r+1}\right)$. This follows from step five, just as step four followed from three.

Finally, $F_{s}\left(m \cdot 2^{r}\right) \subset F_{s}\left(2^{k}\right)$, for any $k$. It suffices to take $k=r$ (by the previous steps). Let $\left\{p_{1}, p_{2}, \ldots, p_{\left(m \cdot 2^{\prime}\right)}\right\}$ be a simple orbit of period $m \cdot 2^{r}$, with $p_{i}<p_{i+1}$, and let

$$
D_{k}=\left[p_{(k-1) m+1}, p_{k m}\right], \quad \text { for } 1 \leq k \leq 2^{r} \text {. }
$$

Then $f$ permutes the intervals $D_{k}$, so there are subintervals $J_{k}$ that (after renumbering) form a loop of length $2^{r}$ as in the lemma. Thus we obtain a periodic orbit of period $2^{r}$, which is simple since the orbit $\left\{p_{i}\right\}$ was. Theorem A now follows immediately.

\section{Stratifications}

The arguments in [4] show that $\mathrm{cl} F(j) \subset$ int $F(k)$, if $k \Delta j$. The only non-trivial case is $k=2^{r}, j=2^{r+1}$, and $f \in \mathrm{cl} F(j)$ with a periodic orbit $\gamma$ of period $k=2^{r}$, the limit of $2^{r+1}$-periodic orbits; it suffices to take $k=2$ and $j=4$ (i.e. $r=1$ ), since we may consider $g=f^{\left(2^{r-1}\right)}$. The derivative of $f^{2}$ on $\gamma$ is negative, so by the implicit function theorem $\gamma$ persists under perturbation of $f$. Therefore $f \in$ int $F(k)$.

In general, the homoclinic and periodic classifications do not mesh well [5]: the best that can be said is that

$$
H\left(2^{r}\right) \subset H\left(2^{r+1}\right) \cup H\left(2^{r+2}\right), \quad \text { for any } r \geq 0,
$$

while for odd $m>1$,

$$
F\left(m \cdot 2^{r}\right) \subset H\left(2^{r}\right) \cup H\left(2^{r+1}\right) \text { and } H\left(2^{r}\right) \subset F\left(3 \cdot 2^{r+1}\right) .
$$

In this section we specialize to a class of maps for which the homoclinic classification gives a stratification, which does mesh nicely with (is refined by) the Sarkovskii stratification. Let $\mathscr{C}$ denote the maps $f \in C^{3}(I, I)$ which have a unique critical point (are 'unimodal') and negative Schwarzian derivative, i.e.

$$
\frac{f^{\prime \prime \prime}}{f^{\prime}}-\frac{3}{2}\left(\frac{f^{\prime \prime}}{f^{\prime}}\right)^{2}<0
$$


whenever $f^{\prime} \neq 0$. We also require that $f(0)=0=f(1)$, and impose the $C^{3}$-topology. This class of maps has been widely studied; see for example [7], [10], [12], [14].

TheOREM B. In $\mathscr{C}, F\left(m \cdot 2^{r}\right) \subset H\left(2^{r}\right)$, for any odd $m>1$ and $r \geq 0$.

Proof. Let $f \in F\left(m \cdot 2^{r}\right)$, and let $P$ be a simple orbit of period $m \cdot 2^{r}$, with blocks $P_{1}, P_{2}, \ldots, P_{\left(2^{r}\right)}$ of $m$ consecutive points as before. We may assume $m \geq 7$, by the results of $\S 2$.

Let $l_{i}$ and $\rho_{i}$ denote the least and greatest points of $P_{i}$; then the unique critical point $c$ of $f$ satisfies

$$
l_{k} \leq c<l_{k+1},
$$

for some $k, 1 \leq k<2^{r}$. Set $g=f^{\left(2^{r}\right)}$ and $P_{k}=\left\{p_{1}, \ldots, p_{m}\right\}$ where $g\left(p_{i}\right)=p_{i+1}$. $P_{k}$ is simple under $g$, so

$$
p_{m}<p_{m-2}<\cdots<p_{1}<p_{2}<\cdots<p_{m-1}
$$

or the opposite order; for definiteness, we consider the order given. Let

$$
I_{1}=\left[p_{1}, p_{2}\right], \ldots, I_{m-1}=\left[p_{m}, p_{m-2}\right]
$$

as in the usual Markov graph for $\left\{p_{k}\right\}$ and $g$, and note that $g$ is somewhere increasing in $I_{m-1}$, somewhere decreasing in $I_{m-3}=\left[p_{m-2}, p_{m-4}\right]$, so that $c \in I_{m-1} \cup I_{m-3}$; (otherwise, $f$ would be monotone on $I_{m-1} \cup I_{m-3}$; since by choice of $k, f$ is monotone on each interval $\left[l_{i}, \rho_{i}\right]$ for $i \neq k$, it would follow that $g$ is also monotone on $I_{m-1} \cup I_{m-3}$, a contradiction).

Since $c$ does not lie between $f^{i}\left(p_{1}\right)$ and $f^{i}\left(p_{2}\right)$ for $0 \leq i \leq 2^{r+1}$, there exists an interval $J \subset I_{1}$ such that $g(J) \subset I_{1}$ and $g^{2}$ is a homeomorphism of $J$ onto $I_{1}$. Note that $g$ must be monotonically decreasing, and $g^{2}$ monotonically increasing, on $J$. Thus $g$ has a fixed point $q \in J$. Since $c$ cannot be in the semi-local stable manifold of any fixed point of $g^{2}$ in $J$, there can be no sink in $J$, [14]. Since $g^{2}$ is a homeomorphism on $J$, there must be a sink between any two fixed sources. Hence $q$ is a source, and the only fixed point of $g^{2}$ in $J$. Therefore $J \subset W^{u}(q, g)$ and

$$
I_{m-1} \subset g^{m+2}(J) \subset W^{u}(q, g) .
$$

But $q \in g\left(I_{m-1}\right)$, so $q$ is a homoclinic fixed point of $g$, whose period under $f$ is $2^{r}$. Thus $F\left(m \cdot 2^{r}\right) \subset H\left(2^{r}\right)$.

We remark that the assumption of negative Schwarzian is necessary in theorem B [5]. Otherwise the orbit of period $2^{r}$ produced above may be contained in an invariant set of intervals on which $f^{\left(2^{\prime}\right)}$ is orientation-reversing. The endpoints will then be a homoclinic orbit, but of period $2^{r+1}$.

TheOREM C. In $\mathscr{C}, H\left(2^{r}\right)$ is a closed set.

Proof. Let $f_{n}$ be a sequence in $\mathscr{C} \cap H\left(2^{r}\right)$ converging to $f$, let $s=2^{r}$, and let $g_{n}=\left(f_{n}\right)^{s}$, $g=f^{s}$; then $g_{n} \rightarrow g$ and it suffices to show that $g$ has a homoclinic fixed point. We may assume that each $g_{n}$ is orientation-reversing at its homoclinic fixed point $p_{n}$ (or else, [5], each has a point of period three and we are done). Also $p_{n} \rightarrow p=g(p)$, and a sequence $z_{k, n}$ in $W^{u}\left(p_{n}, g_{n}\right)$ exists, converging to $p_{n}$ as $k \rightarrow \infty$, with

$$
g_{n}\left(z_{0, n}\right)=p_{n} \text { and } g_{n}\left(z_{k+1, n}\right)=z_{k, n}
$$


Finally, let $z_{k}=\lim _{n} z_{k, n}$, so

$$
g\left(z_{0}\right)=p \text { and } g\left(z_{k+1}\right)=z_{k} .
$$

Note that $\left(g_{n}\right)^{\prime}\left(p_{n}\right) \leq-1$ implies $g^{\prime}(p) \leq-1$; also that $\left(g_{n}\right)^{\prime}$ is positive at some point between $z_{0, n}$ and $z_{2, n}$, so $z_{0} \neq p$ and the $z_{k}$ are distinct. At $f^{n}(p)$ one may construct corresponding points $\left\{z_{k}(n)\right\}$, such that $g\left(z_{k+1}(n)\right)=z_{k}(n)$ and $g\left(z_{0}(n)\right)=f^{n}(p)$, and the points $z_{k}(n)$ occur on each side of $f^{n}(p)$.

Since $f \in F\left(3 \cdot 2^{r+1}\right), p$ must be a (topological) source. Otherwise, the critical point is in the semi-local stable manifold of $f^{k}(p)$, for some $k \leq 2^{r}$ [14]. This orbit is simple (or $f \in F\left(3 \cdot 2^{r-2}\right) \subset H\left(2^{r}\right)$, [2]), and $f$ is a homeomorphism on each component of $I \backslash \bigcup_{k} \operatorname{slsm}\left(f^{k}(p)\right)$, so every period of $f$ must factor $2^{r}$, a contradiction.

Suppose that $W^{u}(p, g)$ contains no critical points of $g$. The two endpoints of $W^{u}(p, g)$ are then not in $W^{u}(p, g)$, but form a periodic orbit of period two under $g$, which is stable on at least one side (i.e. the side towards $p$, contained in $W^{u}(p, g)$ ). This orbit's stable manifold contains a critical point, so it cannot be stable on just the side overlapping $W^{u}(p, g)$; but then we have a sink of period $2^{r+1}$, and (as above) either $f \in H\left(2^{r}\right)$ or every period factors $2^{r+1}$, a contradiction. Hence $W^{u}(p, g)$ does contain a critical point of $g$. It follows that $W^{u}\left(f^{n}(p), g\right)$ contains the critical point $c$ of $f$, for some $n$, and that $W^{u}\left(f^{n+1}(p), g\right)$ contains a point $x$ such that

$$
g(x)=f(c)=\sup (\text { Range }(f)) \text {. }
$$

Therefore $W^{u}\left(f^{n+1}(p), g\right)$ contains the points $z_{k}(n+1)$, and $f^{n+1}(p)$ is homoclinic. Thus $f \in H\left(2^{r}\right)$, and $H\left(2^{r}\right)$ is closed.

THEOREM D. In $\mathscr{C}, H\left(2^{r}\right)=\mathrm{cl} \bigcup_{m \text { odd }} F\left(m \cdot 2^{r}\right)$.

Remark. The previous theorems show that

$$
\operatorname{cl} \cup F\left(m \cdot 2^{r}\right) \subset \operatorname{cl} H\left(2^{r}\right)=H\left(2^{r}\right),
$$

so we need only show that $H\left(2^{r}\right) \subset \operatorname{cl} \bigcup F\left(m \cdot 2^{r}\right)$; this latter statement is true in general, i.e. in $C^{k}(I, I), 1 \leq k \leq \infty$, as will be clear from the following.

Proof. Let $f \in H\left(2^{r}\right)$ and $g=f^{\left(2^{\prime}\right)}$. We may assume a point $p$ of period $2^{r}$ under $f$, with $g^{\prime}(p) \leq-1$, and sequences $z_{2 n}, z_{2 n+1}$, as before. Let $j$ be the smallest positive integer such that $f^{j}\left(z_{0}\right)$ is in the orbit of $p$, and let $k=j-1$. Let $\delta$ be a $C^{\infty}$-small non-negative bump function supported in a neighbourhood $U$ of $f^{k}\left(z_{0}\right)$ such that $U$

(i) contains no point in the orbits of $p$ or any of the $z_{n}$ (except of course $f^{k}\left(z_{0}\right)$ itself), and

(ii) contains no critical point of $g$, unless $f^{k}\left(z_{0}\right)$ is itself such a point, in which case we require $\delta$ to be flat on a still smaller neighbourhood of $f^{k}\left(z_{0}\right)$.

Finally, let $\tilde{f}=f \pm \delta$, where the sign is chosen so that $\tilde{g}^{n}\left(z_{0}\right)<p$. Then $\tilde{g}^{n}\left(z_{0}\right)=z_{2 n}$, for appropriate small $\delta$ and some large $n$, and $z_{2 n}$ is a periodic point of $\tilde{g}$, of period $2 n+1$, and $f \in F\left((2 n+1) \cdot 2^{r}\right)$. Thus $H\left(2^{r}\right) \subset \mathrm{cl} \bigcup_{m} F\left(m \cdot 2^{r}\right)$.

THEOREM E. Any map in a $C^{0}$-neighbourhood of a map with a homoclinic point of period $2^{r}$ has a point of period $3 \cdot 2^{r+1}$; i.e.

$$
H\left(2^{r}\right) \subset \text { int } F\left(3 \cdot 2^{r+1}\right) .
$$


Proof. It suffices to assume $r=0$, and again to take the orientation-reversing case. Then

$$
z_{0}<z_{2}<z_{4}<p<z_{5}<z_{3}<z_{1}
$$

so

$$
f^{6}\left(z_{5}\right)<z_{5}<f^{2}\left(z_{5}\right)<f^{4}\left(z_{5}\right)
$$

This condition implies that $f^{2}$ has a point of period three, and persists under perturbations of $f$, so any $g$ near $f$ is in $F(6)$.

\section{REFERENCES}

[1] L. Block. Homoclinic points of mappings of the interval. Proc. Amer. Math. Soc. 72 (1978), 576-580.

[2] L. Block. Simple periodic orbits of mappings of the interval. Trans. Amer. Math. Soc. 254 (1979), 391-398.

[3] L. Block. Stability of periodic orbits in the theorem of Sarkovskii. Proc. Amer. Math. Soc. 81 (1981), 333-336.

[4] L. Block \& D. Hart. The bifurcation of periodic orbits of one-dimensional maps. Ergod. Th. \& Dynam. Syst. 2 (1982), 125-129.

[5] L. Block \& D. Hart. The bifurcation of homoclinic orbits of maps of the interval. Ergod. Th. \& Dynam. Syst. 2 (1982), 131-138.

[6] L. Block, J. Guckenheimer, M. Misiurewicz \& L.-S. Young. Periodic points and topological entropy of one dimensional maps. Lect. Notes Math. 819 (Springer, 1980), 18-34.

[7] P. Collet \& J.-P. Eckmann. Iterated maps on the interval as dynamical systems, Prog. Phy. 1 (Birkhauser, 1980).

[8] C.-W. Ho. On the structure of the minimum orbits of periodic points for maps of the real line. Preprint.

[9] L. Jonker. Periodic orbits and kneading invariants. Proc. London Math. Soc. 39 (1979), 428-450.

[10] O. Lanford. Smooth transformations of intervals, Lect. Notes Math. 901 Springer (1981), 36-54.

[11] M. Misiurewicz. Horseshoes for mappings of the interval. Bull. Acad. Polon. Sci. 27 (1979), 167-169.

[12] M. Misiurewicz. Structure of mappings of an interval with zero entropy, Publ. Math. IHES, to appear.

[13] A. N. Sarkovskii. Coexistence of cycles of a continuous map of the line into itself, (Russian) $U k r$. Mat. Z. 16 (1964), 61-71.

[14] D. Singer. Stable orbits and bifurcation of maps of the interval, SIAM J. Appl. Math. 35 (1978), 260-267.

[15] P. Stefan. A theorem of Sarkovskii on the coexistence of periodic orbits of continuous endomorphisms of the real line. Comm. Math. Phys. 54 (1977), 237-248. 